\title{
Novel Methods for Human-Computer Interaction in Multimodal and Multidimensional Noninvasive Medical Imaging
}

\author{
Tomasz Soltysinski \\ Institute of Precision and Biomedical Engineering, Warsaw University of Technology, \\ Sw. A. Boboli 8, 02-525 Warsaw, Poland \\ solek@mchtr.pw.edu.pl
}

\begin{abstract}
The newly developed method for medical noisy data segmentation for the purpose of presentation and supporting the diagnostics is introduced. It also allows for morphometry and visualization of medical multimodal and dynamical data. A general mathematical framework is presented and characterized together with numerous applications. As this tool is designed to support human-computer interaction by means of involving the sense of sight, and suspected to be worthy in the virtual environment sensitive to the sense of touch, the discussion is supported with numerous examples of visualizations and multimodal and multidimensional applications of proposed method.
\end{abstract}

Keywords: Digital human modeling, Bayesian inference, spectral method, multidimensional imaging, ultrasound, CT, MRI, PET, histopathology.

\section{Introduction}

Medical imaging is one of the most important fields of present days science. This field is based on numerous achievements of mankind technology and science done in recent decades. It requires fast computers, modern high quality materials and specialized electronic circuits of high speed together with sophisticated software. However, the human factor is still the crucial point of the diagnostic process. Even if the equipment is sophisticated, the decision that physician makes is based on his experience and knowledge as well as the information which he gains during the medical imaging.

Very effective and fast imaging equipment provide enormous amount of data, often in distributed manner. This data covers dynamical time series of signals, complimentary spatial data of images and volumes, as well as some other data that is related to individual patient history and condition. All the data is stored by computers and these machines are responsible for its presentation to the physicians, researchers and the patients as well. This indicates the strong need for methods of data presentation, analysis and visualization.

Throughout this study, some new trends are presented and methods applied to different modalities of medical imaging. This is followed by discussion of the 
possible ways of data combination and presentation to the end users of modern complex and synergistic medical systems.

\section{Methods}

The main point is to focus on multimodal imaging and visualization of data in 3-, 4-, or N-dimensional space. Combining this methodology with high level math and data presentation technics is believed to broaden the view on medical diagnostic process. A special attention is paid to combination of multimodal data by means of Bayesian inference. Medical imaging is a process that transforms the information about the body state into some virtual numerical space. This transformation depends on many circumstances and parameters, hence the final result is known only with some probability dependent on these parameters. Some models may also be adapted into the imaging, however their incorporation requires to use the terms of probability as well. Then, the imaging is just the manipulation of probabilities and the search for a strategy that maximizes the probability that the correct and the most relevant information is obtained.

The proposed method is based on Bayesian inference that provides the information about edges of the investigated structure or organ. This information is fuzzy and uncertain and forms radial function of initial guess on edge position, called edge map. This edge map is used as initial data for spectral method that reveals the final radial function describing the most probable contour of the structure. Important point of proposed method is the incorporation of priors. The a priori knowledge is provided by patterns or probability distributions into the inference. The priors are constructed of denoised and decomposed data. The decomposition is done in multiscale fashion, by means of a trous, B3-spline wavelet based decomposition of images. The priors are also constructed from analytical functions when necessary, if these functions may be guessed or approximated. Application of Bayesian inference and the usage of priors handles with the cases that are hard to analyze and delineate, like those with high level of noise or distortion.

\section{Multidimensional Segmentation}

The proposed method is a new approach to image segmentation that combines the speed of spectral method in contour detection [5] and the Bayesian inference that allows for the most probable estimation on initial edge map. The method has been successfully applied to ultrasonic data and CT brain data with aneurysm and is described in details in $[3,4]$. The real contour of investigated and segmented organ [4] is extracted in the iterative process of solving the nonlinear partial differential equation (PDE). This step is realized in Fourier space by fast spectral method. PDE is approaching the function that reveals the real edge of the object and starts from an initially guessed edge map. This iterative process is controlled by two parameters that describe fidelity of reconstruction, one, $\mu$ is steering the PDE and the other is responsible for slight smoothing of the resolved subsequent partial approximations of the final solution. 


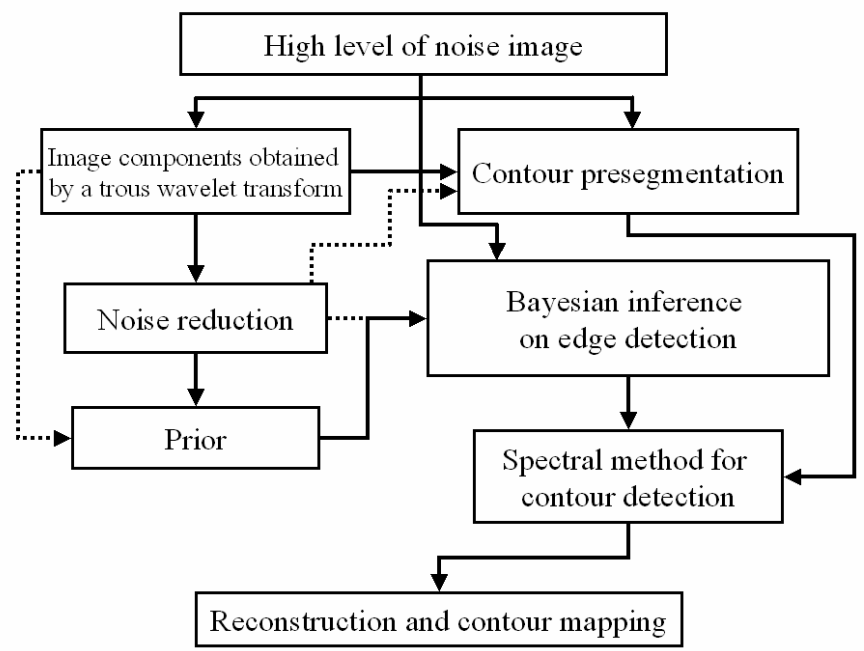

Fig. 1. Flow diagram for proposed Bayesian constrained spectral method. Solid lines mark necessary steps in the algorithm while dotted lines optional paths.

Contouring may be expressed as partial differential equation (PDE). This is the approach commonly found in methods based on active contours that are iteratively approaching the final contour. Following the work presented in [5] the final contour $f$ may be found by solving the elliptic equation of Helmholtz type

$$
\nabla^{2} f-\mu(f-g)=0
$$

This equation uses known variable which is initially guessed edge map $g$. It is solved in spherical coordinates. Moving the $g$ term to right hand side and relating it to a previously found value of $f$, called $f_{n}$ the PDE can be expressed in linearized form

$$
\alpha \nabla^{2} f_{n+1}-f_{n+1}=g_{f_{n}}
$$

Such an equation is further easily solved by the fast spectral method. Applying $\alpha=1 / \mu$ the solution may be controlled by value of $\mu$.

\section{Bayesian Inference in Tissue and Shape Selection}

The edge map $g$ is determined by Bayesian inference on edge placement in image data. Let $P\left(E_{i} / I\right)$ denote the required probability of the most appropriate edge in our existing data set. This is the conditional probability as it depends on the contents of $I$. $P\left(E_{i} I I\right)$ is the probability of the fact that the $I$ point belongs to the edge class $E_{i}$, knowing the value of intensity of this point. Let $P\left(I / E_{i}\right)$ be a probability of how much the value or intensity of a point is depending on edge class $E_{i}$. This term serves as a kernel. $P\left(E_{i}\right)$ is simply the probability of existence of the edge class $E_{i}$ among all other 
detected edge classes. Edge class is a set of some subsequent pixel intensity values. Then the required probability can be found by solving the Bayes rule:

$$
P\left(E_{i} / I\right)=\frac{P\left(I / E_{i}\right) P\left(E_{i}\right)}{P(I)}=\frac{P\left(I / E_{i}\right) P\left(E_{i}\right)}{\sum_{i} P\left(I / E_{i}\right) P\left(E_{i}\right)}
$$

$P(I)$ is a sum of all probabilities $P\left(I / E_{i}\right)$ weighted by $P\left(E_{i}\right)$ and thus remaining constant. $P(I)$ is only a normalizing term and can be excluded from further analysis. The standard way of solving Bayes equation is the maximization of the right side over the parameter $E_{i}$ (maximum likelihood, $M L$ step) and then maximization of the found solution over all accessible data (maximum-a-posteriori, MAP step). The $P\left(E_{i}\right)$ is a prior and contains a priori knowledge.

In practice the $P\left(I / E_{i}\right)$ is estimated from the histogram of $I$ along given radius from centroid to a point of circumference of some bounding box selecting the region of interest $(R O I)$. The histogram is shrank in such a way that each bin is equal to the edge class size assuming that each expected edge class covers the same number of intensity levels. Calculating the normalized probability over all edges $E_{i}, M L$ step is performed and the most probable edge in $I$ is estimated. Then the MAP is done by searching for maximum over the data itself, and usually the first maximum in $P\left(I / E_{i}\right)$ is detected as an edge. $P\left(E_{i}\right)$ may be a constant value if we assume all edge classes as equally probable or may be distributed uniquely according to the prior knowledge. From $P(E / I)$ the polar edge map, $g(\theta)$ is derived.

\section{Spectral Methods}

The linearized equation 2 is solved by a spectral method [5]. Adapting Cheong's method [11] the Laplacian operator $\nabla^{2}$ on the unit circle is expressed as follows:

$$
\nabla^{2} \frac{1}{\sin \theta} \frac{\delta}{\delta \theta}\left(\sin \theta \frac{\delta}{\delta \theta}\right)
$$

Both functions, $f$ and $g$ are defined on the computational grid $\left(\theta_{i}\right)$, where $\theta_{i}=\pi(j+0.5) / J$. $J$ is the number of points along the longitude of unit circle's circumference high enough to engage all points covered by $g$. Each point in $g$ may be now expressed by its discrete cosine transform (DCT) representation yielding

$$
g\left(\theta_{i}\right)=\sum_{n=0}^{J-1} g_{n} \cos n \theta_{i}
$$

with $g_{n}$ being simply the coefficients of discrete cosine transform. Applying equation 4 into equation 1, it may be written as an ordinary differential equation (ODE):

$$
\frac{1}{\sin \theta} \frac{\delta}{\delta \theta}\left(\sin \theta \frac{\delta}{\delta \theta} f(\theta)\right)=\mu[f(\theta)-g(\theta)]
$$


which yields an algebraic system of equations in Fourier space:

$$
p_{n-2} f_{n-2}-p_{n} f_{n}+p_{n+2} f_{n+2}=\mu\left[\frac{1}{4} g_{n-2}-\frac{1}{2} g_{n}+\frac{1}{4} g_{n+2}\right]
$$

where

$$
\begin{gathered}
p_{n-2}=\frac{(n-1)(n-2)+\mu}{4}, \\
p_{n}=\frac{n^{2}+\mu}{2}, \quad p_{n+2}=\frac{(n+1)(n+2)+\mu}{4}
\end{gathered}
$$

after substitution of eq. 5 into eq. 6 and expressing $f$ in the same way as $g$. The index $n=1,3, \ldots J-1$ for odd $n$ and $n=0,2, . ., J-2$ for even $n$. The system of equation 7 may be now expressed as a double matrix equation:

$$
\mathbf{B}_{\mathrm{e}} \hat{\mathrm{h}}_{\mathrm{e}}=\mathbf{A}_{\mathrm{e}} \hat{\mathrm{g}}_{\mathrm{e}}, \quad \mathbf{B}_{\mathrm{o}} \hat{\mathrm{h}}_{\mathrm{o}}=\mathbf{A}_{\mathrm{o}} \hat{\mathrm{g}}_{\mathrm{o}}
$$

with subscripts $e$ for even and $o$ for odd $n, \hat{h}$ and $\hat{g}$ denote the column vector of expansion coefficients of $f(\theta)$ and $g(\theta)$, respectively. B is a tridiagonal matrix containing the left side of equation 7 and $\mathbf{A}$ is tridiagonal matrix with constant coefficients along each diagonal corresponding to right side of eq. 7.

The calculated set of expansion coefficients $f_{n+1}$ serves for the reconstruction of $f_{i}$ the representation of $g$ on the certain level of approximation $i$. Smoothing and summing all partial functions $f_{i}$ the required smooth approximation to $g$ is recovered revealing the most probable edge map.

\section{Wavelet a trous Analysis}

It may be summarized as follows:

1. Initialize $\mathrm{j}$, the scale index, to 0 , starting with an image $c_{j, k}$ where $\mathrm{k}$ ranges over all pixels.

2. Carry out a discrete convolution of the data $c_{j, k}$ using a wavelet filter, yielding $c_{j+1, k}$. The convolution is an interlaced one, where the filter's pixel values have a gap (growing with level $\mathrm{j}$ ) between them of $2^{\mathrm{j}}$ pixels, giving rise to the name $a$ trous - with holes.

3. From this smoothing it is obtained the discrete wavelet transform, $w_{j+1, k}=c_{j, k^{-}}$ $c_{j+l, k .}$

4. If $\mathrm{j}$ is less than the number $\mathrm{J}$ of resolution levels wanted, then increment $\mathrm{j}$ and return to step 2 .

The original image is reconstructed by the summation of all $w_{j, k}$ and the smoothed array $c_{J, k}$, where $\mathrm{J}$ is the maximum that $\mathrm{j}$ may reach.

For the purpose of this study, further in the paper, the reversed notation is used that, as it is believed, makes the analysis more intuitive and convenient. The smoothed component (originally $c_{J, k}$ ) has index 0 and all subsequent components, with increasing level of details have growing indexes. Hence, level $w_{l, k}$ (originally $w_{J, k}$ ) 


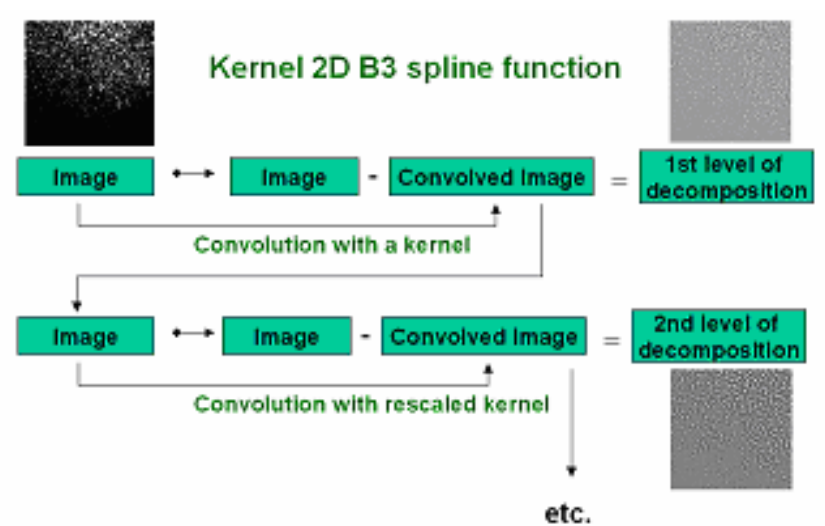

Fig. 2. Scheme that describes a trous wavelet decomposition algorithm

with index 1 has low details but of higher resolution than the base level with index 0 , level $w_{2, k}$ contains details with higher resolution than previous level but lower than these ones at next level, etc.

\section{Practical Applications for Human-Computer Interaction}

\section{Hierarchical, Dynamical and Structural Modeling}

The method has been tested in many scales from cell level through internal organs to whole body. This makes it a universal tool for segmentation of a human tissue and organs of body in hierarchical or dynamical digital models. Another advantage of the method is arbitrary scaling of surface of visualized object and compression of information which is necessary for object's description. Applications of this approach are shown for delineation of heart left ventricle walls in dynamic ultrasonic imaging at high level of speckle noise (figs. 3,4) together with derivation of unique proposal for dynamical analysis based on time-contour function plane (fig. 5) covering the total information about dynamics of shape of heart left ventricle; morphometry and visualization of upper part of a thigh bone (figs. 6,7) compared to method based on pixel counting; delineation of active metabolic region of liver in PET-CT multimodal
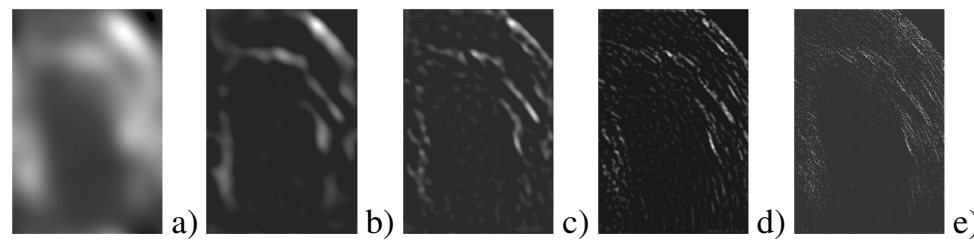

Fig. 3. Multiscale a trous decomposition of heart left ventricle ultrasonic image of one frame of cardiac cycle. Scales of decomposition, from grain level (a) to finer scales (b,c,d,e). 

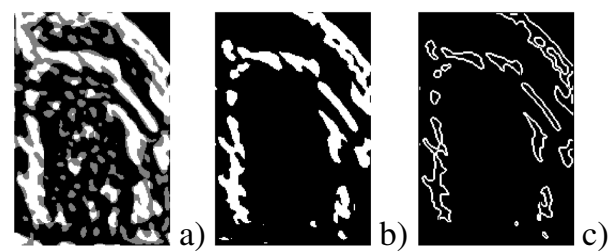

Fig. 4. Construction of enhanced prior for improved strategy in Bayesian inference based on multiresolution support (MRS), a technics based on a trous multiscale decomposition. Original image of MRS (a), filtered image (b) and the same image with edges extracted by Sobel filtration (c), the final prior used in inference.
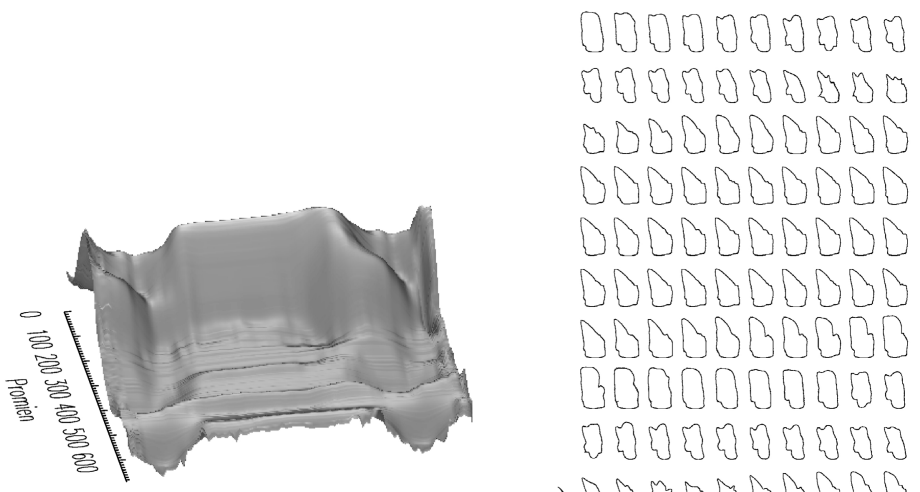

a) $D, 30 \square 0 B \Omega 0 B$ b)

Fig. 5. Time-contour radial function space (a). The dynamics of mitral valve region is visible. All the cycle contours derived in fully automatic manner from raw, ultrasonic image data (b).
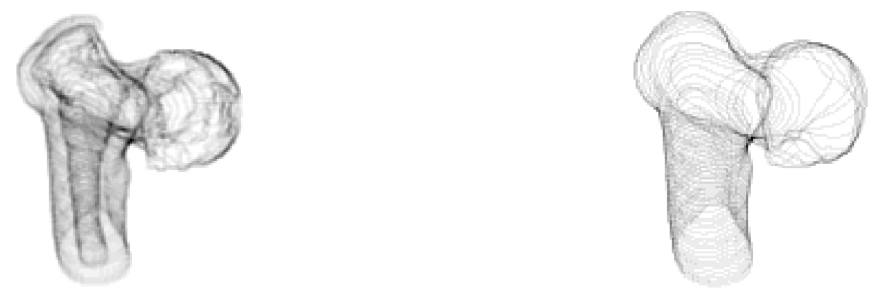

a)

b)

Fig. 6. Comparison of contours in 3D structure of a piece of thigh bone obtained by Sobel edge detection (a) and proposed method based on Bayesian inference (b)

parallel imaging (figs. 8,9) with the applications of complex priors for Bayesian inference; morphometry of structural and functional regions of brain tumor tissue in subsequent MRI-PET whole head scans (fig. 10) and in cells morphometry for angiogenesis research (fig. 11). 
a)

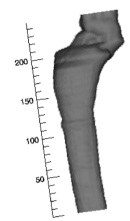

b)

Fig. 7. Examples of the bone shown on fig. 6 reconstructed by proposed method and visualized in IDL environment
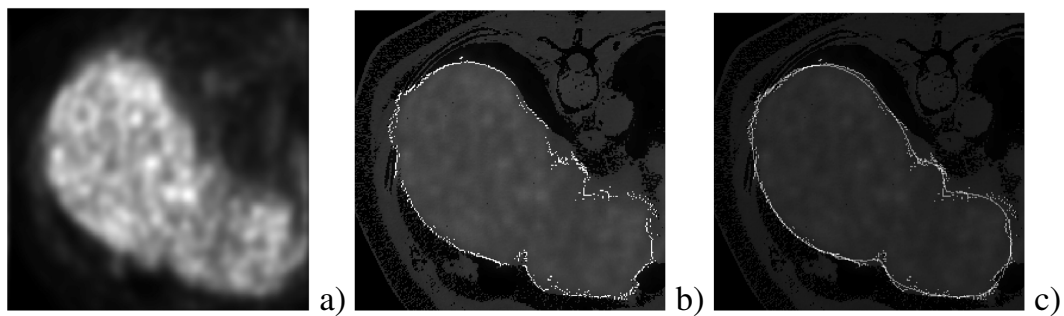

Fig. 8. The prior for Bayesian inference on the region of PET activity in human liver (a). Initial edge map revealed by Bayesian inference (b) and the resulting contour based on this map, returned by spectral method (c).
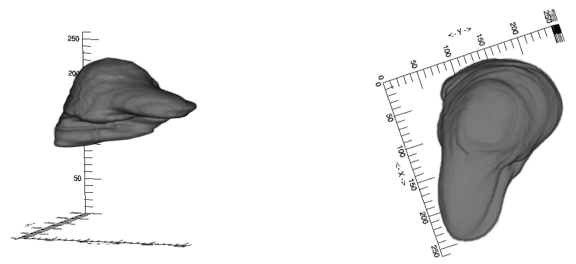

a)

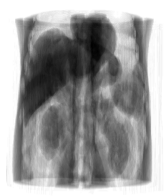

b)

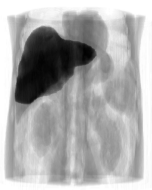

d) e)

Fig. 9. Visualizations of 3D structure PET active region in human liver determined by proposed method (a,b). Structural 3D projection based on the CT volume (c), functional 3D projection of PET active region of liver obtained by proposed method (d) and the fusion of two projections (e). 
a)

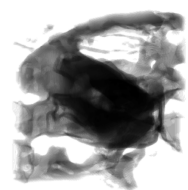

b)

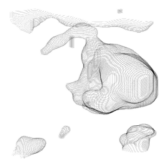

c)

Fig. 10. Fusion (a) of structural MRI (b) and functional PET 3D volumes (c) limited to the tissue of brain cancer. The data was registered first by a method based on mutual information estimation.

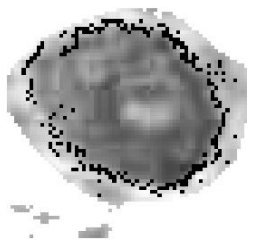

a)

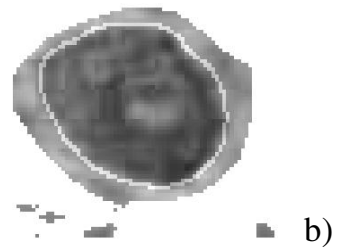

Fig. 11. Cell morphometry for angiogenesis research. Proposed method is applied to find out the edge map of a cell (a) and the final contour (b). Multiscale priors were used.

\section{Discussion}

The method is well suited for the analysis of noisy and disturbed data like that obtained by ultrasonography. Applications of the method to ultrasonic data is an alternative for existing methods [13][14] especially in the case of dynamical modeling. Moreover, it offers a unique opportunity for missing information reconstruction. This is the main advantage of the method in applications to multimodal data as well. High ability of mutual information recovering supports the medical diagnostic process based on human-computer interaction.

\section{Conclusion}

Presented work may serve as a general framework to develop useful and suitable graphical interface for supporting the diagnostic process, it helps to manage huge amount of information about human body and its functions, provides tools for virtual reality and digital human modeling.

Acknowledgments. This scientific work has been funded by resources of Science and High Education Ministry of Poland for years 2006-2007, in the frame of research grant number N518 023 31/1387.

\section{References}

1. Rogowska, J.: Overview of fundamentals of medical image segmentation. In: Bankman, w I.N. (ed.) Handbook of Medical Imaging, Processing and Analysis, pp. 69-86. Academic Press, San Diego (2000)

2. Bankman, I.N. (ed.): Handbook of Medical Imaging, Processing and Analysis. Academic Press, San Diego (2000) 
3. Soltysinski, T.: Novel algorithm for active surface reconstruction by Bayesian constrained spectral method. In: Hozman, J., Kneppo, P. (eds.): IFMBE Proceedings, vol. 11. Prague: IFMBE, pp. 4191-4195 (2005)

4. Soltysinski, T., Kałuźynski, K., Pałko, T.: Cardiac ventricle contour reconstruction in ultrasonographic images using Bayesian constrained spectral method. Lecture Notes in Artificial Intelligence Series. Springer, Heidelberg (in press, 2006)

5. Li, J., Hero, A.O.: A fast spectral method for active 3D shape reconstruction. Journal of Mathematical Imaging and Vision 20, 73-87 (2004)

6. Holschneider, M., Kronland-Martinet, R., Morlet, J., Tchamitchian, P.: A real time algorithm for signal analysis with the help of the wavelet transform. In: w Wavelets: TimeFrequency Methods and Phase-Space, pp. 286-297. Springer, Heidelberg (1989)

7. Soltysinski, T., Rodatus, A., Golnik, N., Pałko, T.: Comparison of multiscale entropy of healthy and cancer tissue imaged by optical polarimeter. In: IFMBE Proceedings, vol. 14 (2006)

8. Soltysinski, T.: Speckle Noise Removal By A Trous Decomposition And Thresholdlimited Synthesis In Ultrasonic Cardiac Images. In: Proceedings of the conference Biosignal 2006, Brno Technical University, Czech Republic, vol. 18 (2006)

9. Gravel, P., Beaudoin, G., De Guise, J.A.: A method for modeling noise in medical images. IEEE Transactions on medical imaging 23(10), 1221-1232 (2004)

10. Starck, J.-L., Murtagh, F.: Astronomical image and data analysis. Springer, Berlin (2002)

11. Cheong, H.: Double Fourier series on a sphere: Applications to elliptic and vorticity equations. Journal of Computational Physics 157(1), 327-349 (2000)

12. Soltysinski, T.: Influence of multiscale denoising on Bayesian constrained spectral method in segmentation of noisy medical images. In: IFMBE Proceedings, vol. 14 (2006)

13. Dydenko, I., Friboulet, D., Gorce, J.M., D’Hooge, J., Bijnens, B., Magnin, I.E.: Towards ultrasound cardiac image segmentation based on the radiofrequency signal. Medical Image Analysis 7, 353-367 (2003)

14. Hammoude, A.: Endocardial border identification in two-dimensional echocardiographic images: review of methods. Computers in Medical Imaging and Graphics 32, 181-193 (1998) 